\title{
Remote Operations in der Prozessautomatisierung
}

\section{Treiber und Entwicklungen}

Der aus der Production IT kommende Technology Push sowie der durch den anstehenden demografischen Wandel verursachte Market Pull generieren eine hohe Innovationsrate in der Automatisierungstechnik verfahrenstechnischer Prozesse. Der Beitrag befasst sich mit Remote Operations, also der Hochautomatisierung und Fernsteuerung von Anlagen. Das ist ein Kernthema unter den Aktivitäten im Rahmen von Operational Excellence. Durch diese Entwicklungen und Industrie 4.0 entsteht ein verstärktes Spannungsfeld zwischen Automatisierung und IT.

\section{SCHLAGWÖRTER Operational Excellence / Prozessautomatisierung / Remote Operations / Namur}

\section{Remote Operations in Process Automation Drivers and Development}

Together, the technology push provided by production IT and the market pull resulting from demographic change are generating a high innovation rate for automation technology in process industries. A key topic among Operational Excellence activities is Remote Operations, i.e. the advanced automation and the remote operational control of plants. As a result of such developments and the progress of Industry 4.0, increasing tensions are developing between automation and IT. 


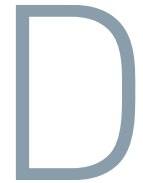
ie Innovationsrate in der Automatisierungstechnik ist hoch. Davon zeugen zahlreiche in der Literatur dargestellte neue Trends, die Produktinnovationen und Neuvorstellungen von Herstellern und nicht zuletzt ein jährliches Marktwachstum von zirka 7 \% [1]. Für diese Entwicklungen gibt es unterschiedliche treibende Faktoren. Zum einen existiert ein deutlicher Technology Push in der Automatisierungstechnik durch die Lösungen, Produkte und Methoden der Production IT: Mittels Adaptionen von Innovationen des ebenfalls sehr neuerungsfreudigen IT-Markts entsteht eine Vielzahl von Lösungen, die in Teilen für die Automatisierungstechnik nutzbar sind. Einige davon haben eine klare Zuordnung zu bereits bestehenden Bedürfnissen, andere suchen noch ihr geeignetes Einsatzfeld.

Ein weiterer Faktor ist der zur Zeit herrschende Market Pull: Grob lässt sich dieser Effekt einteilen in den Wunsch nach Optimierung durch Automatisierung und die Notwendigkeit, Antworten auf die Fragen von demografischem Wandel und gesicherter Wettbewerbsfähigkeit zu finden. Während letztgenannte Aspekte der Startpunkt für Industrie 4.0 sind, mündet der Bedarf an Höherautomatisierung zur wirtschaftlichen Optimierung von verfahrenstechnischen Produktionsstätten in zahlreichen Operational-Excellence-Initiativen der Unternehmen.

Das Ziel von Abschnitt 1 ist es, einen Überblick und Hintergründe zu diesen für die Automatisierung treibenden Faktoren darzustellen. Dabei wird auf die notwendigen Weiterentwicklungen in den Feldern Anlagenautomatisierung, Anlagenintegrität und Visualisierung eingegangen, die für eine effiziente Umsetzung von Remote Operations notwendig sind. In diesem Zusammenhang wird über Erfahrungen bei der BASF berichtet und Anregungen für weitere Entwicklungsschritte gegeben. Das Thema Remote Operations ist Gegenstand von Abschnitt 2.

Letztlich führen die vorgenannten Handlungsfelder wie Remote Operations, Industrie 4.0 und andere innovative Methoden dazu, dass eine stärkere Verschmelzung von Automatisierung und IT notwendig ist. Wegen der ungleichen Größenverhältnisse und Herangehensweisen ist hier ein strukturierter Zugang nötig, um dabei das bestmögliche Ziel für die Produktion erreichen zu können. Entsprechende Ansätze werden im Abschnitt 3 des Beitrags beschrieben. Der Artikel fasst damit die Beiträge der Namur Hauptsitzung $2014 \mathrm{zu}$ Remote Operations und den Konsequenzen verteilter Intelligenz (Namur AK 2.1 PLS und SPS) zusammen.

\section{TREIBER FÜR HÖHERAUTOMATISIERUNG}

In diesem Abschnitt werden Aspekte des Technology Pushs aus der Production IT für die Automatisierungstechnik beleuchtet. Ferner werden die Aspekte des die Automatisierung fördernden Market Pulls erörtert. Dabei geht es um das Ziel der wirtschaftlichen Optimierung von Produktionsprozessen durch eine verbesserte Automatisierung, und es werden die Folgen des demografischen Wandels auf den Stellenwert der Automatisierung behandelt.

\subsection{Production IT als Quelle von Technology Pushes}

Neben den intelligenten Methoden der IT, wie Algorithmen zur Datenkomprimierung, nimmt die Hardwareleistung in den Feldern Rechenleistung, Datenübertragung und Datenspeicherung ständig zu. In Bild 1 sind die exponentielle Steigerung dieser Leistungen gemäß Moore's (Rechenleistung) und Gilder's Law (Datenübertragung) dargestellt. Ein Beispiel für Fortschritte der Netzwerktechnik ist das 5G Lab Germany [4]. Solche Entwicklungen bieten Möglichkeiten für Optimierung und Diagnose.

\subsection{Automatisierung: zunehmend wichtiger Werttreiber}

Die Automatisierung von Prozessen und Anlagen als Ganzes bedient viele Werttreiber der Produktionsun- 


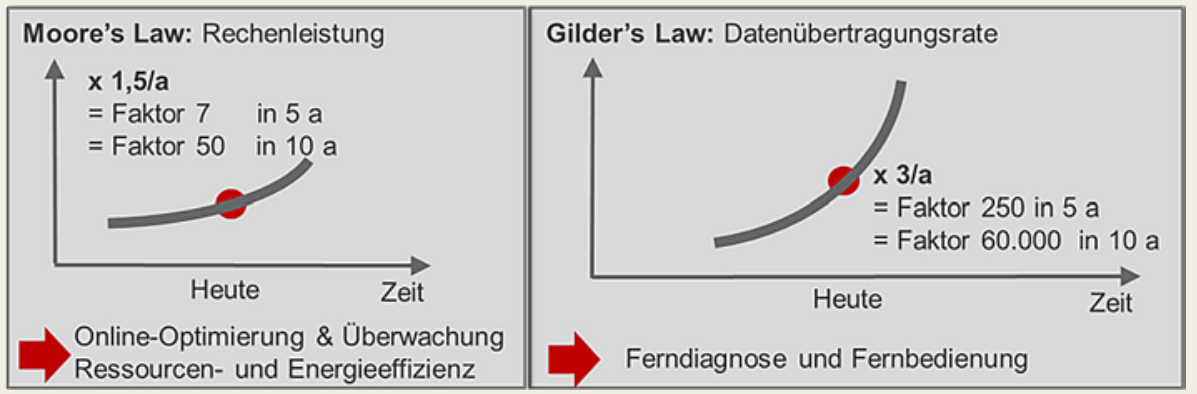

BILD 1: Entwicklung von Rechenleistung und Datenübertragungsrate gemäß Moore's und Gilder's Law.

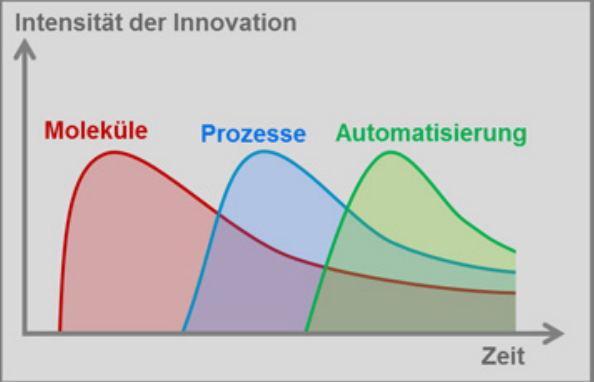

BILD 2: Die Entwicklung der Innovationsintensität in der Prozessautomatisierung

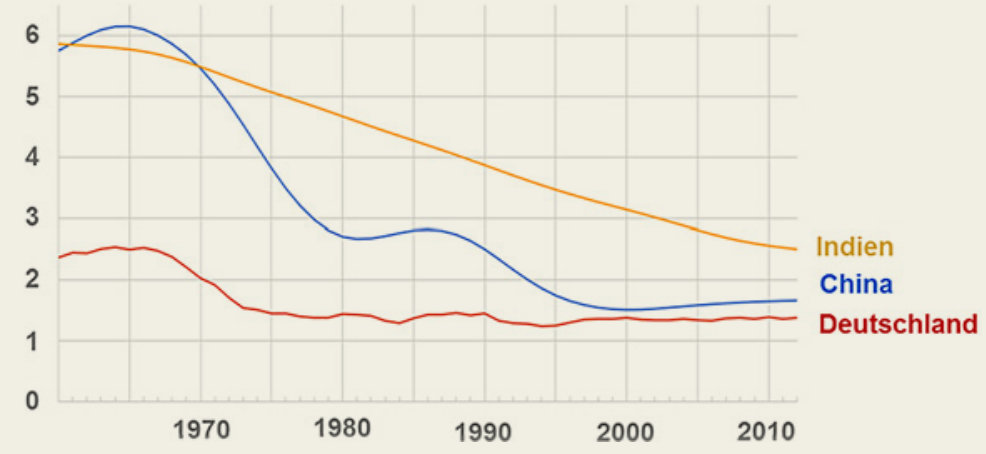

BILD 3: Fruchbarkeitsraten von Indien, China und Deutschland [2] ternehmen, und daraus entsteht naturgemäß der Wunsch, mehr Gewinn durch Automatisierung zu erwirtschaften. Ein Punkt, der sich in der Community der Automatisierer klar herauskristallisiert, ist, dass die Automatisierung verfahrenstechnischer Prozesse klar an Bedeutung in der Gesamtorganisation gewinnt. Für diese Entwicklung gibt es mehrere Einflussfaktoren.

Zum einen ist die Zeit reif dafür. Hierzu bietet Bild 2 eine qualitative Illustration verschiedener Fokusthemen: In den frühen Jahren der Verfahrenstechnik lag die höchste Innovationsintensität klar auf den Molekülen selbst, also auf dem „,was wird produziert?“. Gefolgt wurde dies von einem Fokus auf die Prozesse, also „wie wird produziert?“ Und nun stehen wir vor der Frage: Wie produzieren wir möglichst effizient, was die Automatisierung ins Spiel bringt.

Ein zweiter Faktor ist, dass die Automatisierung als Werttreiber besser erkannt wird, das Marketing funktioniert also in gewissem Sinne besser. Aspekte wie die Beiträge zu Effizienz und Qualität werden nicht in Zweifel gezogen und finden in dem Flaggschiffbegriff Operational Excellence ein neues Zugpferd. Das Fazit aus beiden Aspekten ist, dass die Attraktivität der Automatisierung steigt.

\subsection{Automatisierung: Antwort auf gesellschaftlichen Wandel}

Neben dem Wunsch einer Höherautomatisierung besteht allerdings noch der Zwang, für weitere Fortschritte. Automatisierung ist unbestritten eine Grundlage für den Wohlstand einer Gesellschaft. In der Beobachtung zeigt sich das demografisch-ökonomische Paradoxon: Je mehr Wohlstand eine Gesellschaft erreicht hat, desto geringer ist ihre Geburtenrate. Das löst dann allerdings einen Kreislauf aus: Der Geburtenrückgang führt langfristig dazu, dass der berufsfähige Bevölkerungsanteil sinkt und das wiederum weckt den Bedarf an mehr Automatisierung.

In Deutschland ist uns dieser Effekt intuitiv bewusst. Interessant ist, dass im Rahmen der Globalisierung andere Länder eine ähnliche Entwicklung durchlaufen. Als Beispiele sind in Bild 3 die Fruchtbarkeitsraten, also Geburten pro Frau, für drei Länder gezeigt. Deutschland liegt bereits seit mehreren Jahrzenten unter der Reproduktiongrenze von etwa 2, China hat dies teils durch staatliche Regulierung bereits erreicht und Indien ist auf dem gleichen Weg.

Die Frage, die sich aufdrängt, ist, was sind die zukünftigen Differenzierungsfaktoren für die deutsche Wirtschaft. Auf diese Frage gibt es eine Antwort: In- 
dustrie 4.0 wurde explizit als Programm für den Erhalt der deutschen Wettbewerbsfähigkeit aufgesetzt. Sowohl der Zweck als auch die Lösungen innerhalb von Industrie 4.0 haben eine absolute Daseinsberechtigung, ohne dass sie sich bereits genau scharf definieren ließen. Und dies gilt nicht erst in der Zukunft und bei Neuanlagen, sondern viele Lösungen sind bereits heute einsetzbar.

\section{REMOTE OPERATIONS}

Die Namur adressiert seit Jahren in mehreren Arbeitskreisen das Thema Operational Excellence und Production IT für die Prozessindustrie. In diesem Abschnitt soll der Status hinsichtlich teilautarker Anlagen und Remote Operations zusammenfassend dargestellt werden. Bedarfe zur weiteren Technologieentwicklung ergeben sich aus der notwendigen Reduktion des hohen Implementierungsaufwands. Dazu werden Anforderungen, Lösungsansätze sowie Aufwand/Nutzen-Betrachtungen beschrieben.

In der Prozessindustrie ist die Anwendung von Remote Operations bei der Luftzerlegung bereits weit fortgeschritten. Linde hebt in [5] das neue Remote Operating Center (ROC) hervor, mit dem zentral vom Standort Leuna aus 30 Luftzerlegungsanlagen und 90 ECOVARAnlagen in Deutschland, Österreich, Schweiz und den Benelux-Staaten ferngesteuert werden. Linde senkt dadurch die Energiekosten und erhöht die Betriebssicherheit der Anlagen [5]. Als Basis dient bei Luftzerlegungsanlagen der von der European -Industrial-GasesAssociation (EIGA) im Jahr 2005 erstellte Standard zu „Unmanned Air Gas Plants“ mit dem Fokus auf „Definitionen, Sicherheit, Anlagendesign, Betrieb, Instandhaltung, Notfallpläne“ [6].

\subsection{Entwicklungsgrade}

Bild 4 zeigt verschiedene Entwicklungsgrade von Anlagen in der Prozessindustrie und qualitativ die erforderlichen Investitionssummen beim Übergang in die nächste Stufe.

- Stufe 1: Seit Jahrzehnten werden Anlagen mit Prozessleitsystemen (PLS) ausgestattet und zumindest sukzessive alle Messstellen an das PLS angeschlossen und in ein Plant-Information-Management-System (PIMS) übertragen. Darüber hinaus laufen in den Betrieben seit einigen Jahren Operational-Excellence-Programme beziehungsweise kontinuierliche Verbesserungsprozesse.

- Stufe 2: Eine hochautomatisierte Anlage zeichnet sich unter anderem dadurch aus, dass im Normalbetrieb keine routinemäßigen Bedienereingriffe und manuelle Tätigkeiten erforderlich sind. Das bedeutet insbesondere, dass alle Regelkreise im Automatikbetrieb laufen und die gesamte Anlage ohne Bildwechsel zu überwachen ist. Die Anzahl von Betrieben in dieser Entwicklungsstufe ist noch sehr klein und zeigt Entwicklungsbedarf von Methoden und Werkzeugen zur Unterstützung derartiger Lösungen.

- Stufe 3: Mit noch höherem Aufwand lässt sich eine teilautarke Anlage realisieren, die zumindest auBerhalb der Normalarbeitszeiten unbesetzt ist und durch ein Remote-Operation-Center unterstützt wird. Hierfür ist die betriebsinterne Sicht zu erweitern und eine betriebsübergreifende Entwicklung anzustoßen.

Die Vorteile einer anlagenübergreifenden Steuerung und Überwachung von Prozessanlagen sind:

- Bündelung und Bereitstellung von Expertise zur Qualitätssteigerung der Betreiberaufgaben,

- Verteilung von Aufgaben in einem Technologieverbund zur Steigerung der Flexibilität, beispielsweise durch kompetente Anfahrunterstützung oder bei der Beherrschung von Auslastungsschwankungen

- Betrieb ohne Nachtschicht als Beitrag zur Verbesserung der Sozialverträglichkeit.

Die anlagenübergreifende Aufgabenverteilung kann dabei innerhalb einer Region oder auch innerhalb eines Standorts organisiert werden, sofern dieser hinreichend groß und einheitlich ist. Das bedeutet, dass zum Beispiel bei einem Betrieb ohne Nachtschicht Operateure in einer anderen Leitwarte am Standort oder an einem anderen Standort die Kontrollaufgaben übernehmen. Ein Modell ist, die bisher über die Woche praktisch gleich verteilten Schichtstärken zu ändern und nachts und am Wochenende den Personalaufwand deutlich zu reduzieren und dafür im Gegenzug die Tagschichten zu verstärken, vergleiche Bild 5. Ziel verstärkter Tagschichten ist ein beschleunigter Test und eine schnellere Umsetzung von Optimierungsmaßnahmen, die von Spezialisten ebenfalls in Tagschichten vorgeschlagen werden. Beispiele hierfür sind Prozessoptimierungen zur weiteren kontinuierlichen Produktivitätssteigerung durch Senkung der Energie- oder Rohstoffkosten.

\subsection{Anforderungen und Lösungsansätze}

Neben einer Automatisierungsinfrastruktur für den sicheren Fernzugriff wird in der Praxis die Einheitlichkeit der eingesetzten Lösungen im betrachteten Betriebscluster von Bedeutung sein. Anforderungen bestehen hierbei jeweils für die Prozessautomatisierung sowie die Sicherstellung der Anlagenintegrität.

Prozessautomatisierung: Durch selektive Sensoren und Aktoren sind alle manuellen Tätigkeiten im räumlichen und zeitlichen Bilanzraum zu automatisieren. Dies umfasst die Automatisierung von Last- beziehungsweise Produktwechseln, den Umgang mit Störungen, die Automatisierung von Offline-Analytik 


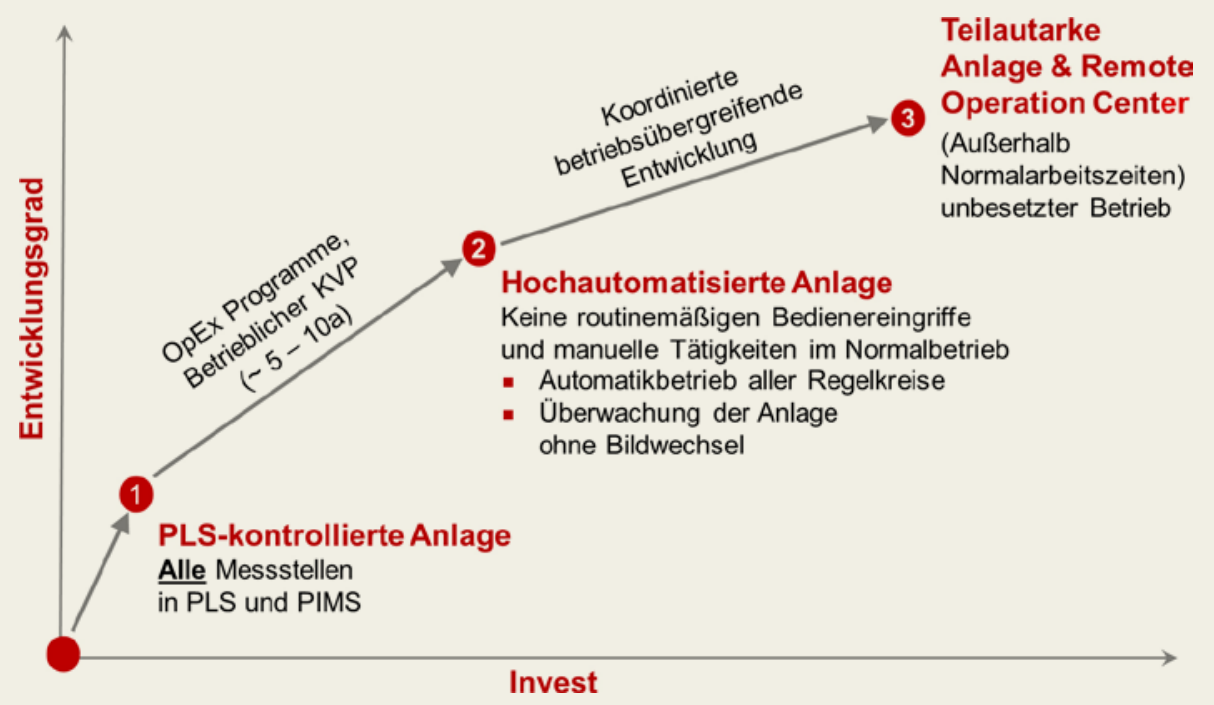

BILD 4: Die Entwicklungsgrade von Anlagen der Prozessindustrie (Außerhalb

Invest

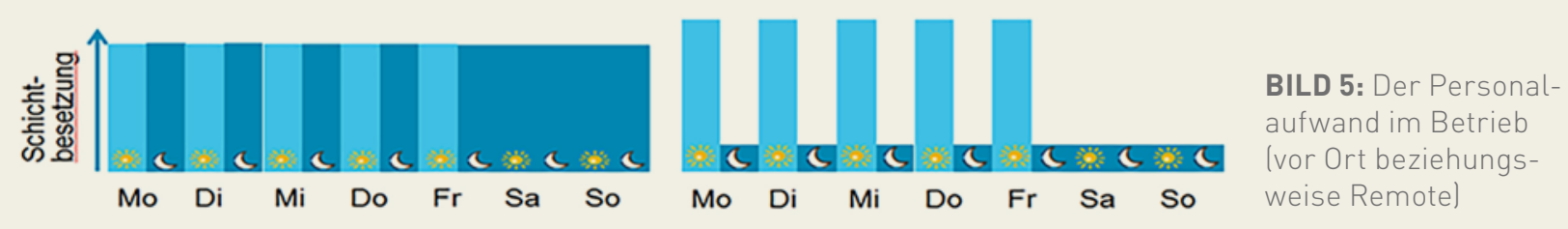

durch Prozessanalysentechnik, die Anlagenbelegungsplanung bis hin zur Logistik. Basis für robuste und stabile Automatisierungslösungen ist dabei eine weitgehende Transparenz über die Prozess- und Anlagenzustände.

Alarmmanagement: Klare Vorgaben zum Alarmmanagement enthält die neue IEC-Richtlinie 62682; im Mittel ist eine maximale Alarmzahl von sechs Alarmen pro Operator und Stunde einzuhalten. Wenn beispielsweise nachts und am Wochenende im Mittel nur ein Operator für zwei Betriebe zur Verfügung steht, liegt der Grenzwert in dieser Zeit bei drei Alarmen/Stunde und Betrieb.

Um diese Anforderung zu erreichen, ist ein gezieltes Alarmengineering erforderlich, bei dem bewusst zwischen Information und Alarmen unterschieden wird. Außerdem kann ein kontinuierliches Alarmmonitoring die Erkennung häufiger Ursachen von Alarmen unterstützen, um zum Beispiel durch eine Höherautomatisierung Alarme an der Ursache zu eliminieren. Darüber hinaus ist es vorteilhaft, zustands- beziehungsweise tageszeitbasiert Alarme zu generieren, um aufkommende Probleme in der Tagschicht zu erkennen und prädiktive Maßnahmen einzuleiten. Entwicklungsbedarf besteht in einem zielgruppenorientierten Werkzeug zur Kausalitätserkennung von Alarmfluten und eventuell einer darauf aufbauenden Unterdrückung nicht sicherheitsrelevanter Folgealarme.

Mensch-Maschine-Schnittstelle: Die Anforderung besteht darin, die Anlagen mit typischerweise mehreren tausend Messstellen stark verdichtet zu charakterisieren. Anstatt der stark verbreiteten Fülle digitaler Information bietet sich durch grafische Aufbereitung die gezielte Nutzung der visuellen menschlichen Fähigkeiten an. Dies bedeutet, Information weitgehend zu visualisieren, zum Beispiel durch Profile, Trends oder Spinnendiagramme. Sehr hilfreich sind dabei die gleichzeitige Darstellung der jeweiligen Akzeptanzbereiche für die einzelnen Größen, die entweder modelloder erfahrungs- beziehungsweise datenbasiert bestimmt werden können, vergleiche Bild 6.

Ein Entwicklungsbedarf zur Bestimmung geeigneter Akzeptanzbereiche besteht insbesondere für BatchProzesse: Neben dem nichtlinearen Prozessverhalten besteht die Herausforderung dabei in häufig wechselnden und immer wieder neuen Rezepturen.

Prozessstabilität: Robust zu automatisieren sind Produktwechsel sowie Schritte von gestörten Zuständen in den Normalzustand. Basis für derartige sequenzbasierte Automatisierungen ist die IEC 61512 für BatchProzesse und die derzeit in Ausarbeitung befindliche ISA SP 106 für kontinuierlich betriebene Prozesse. Ins- 

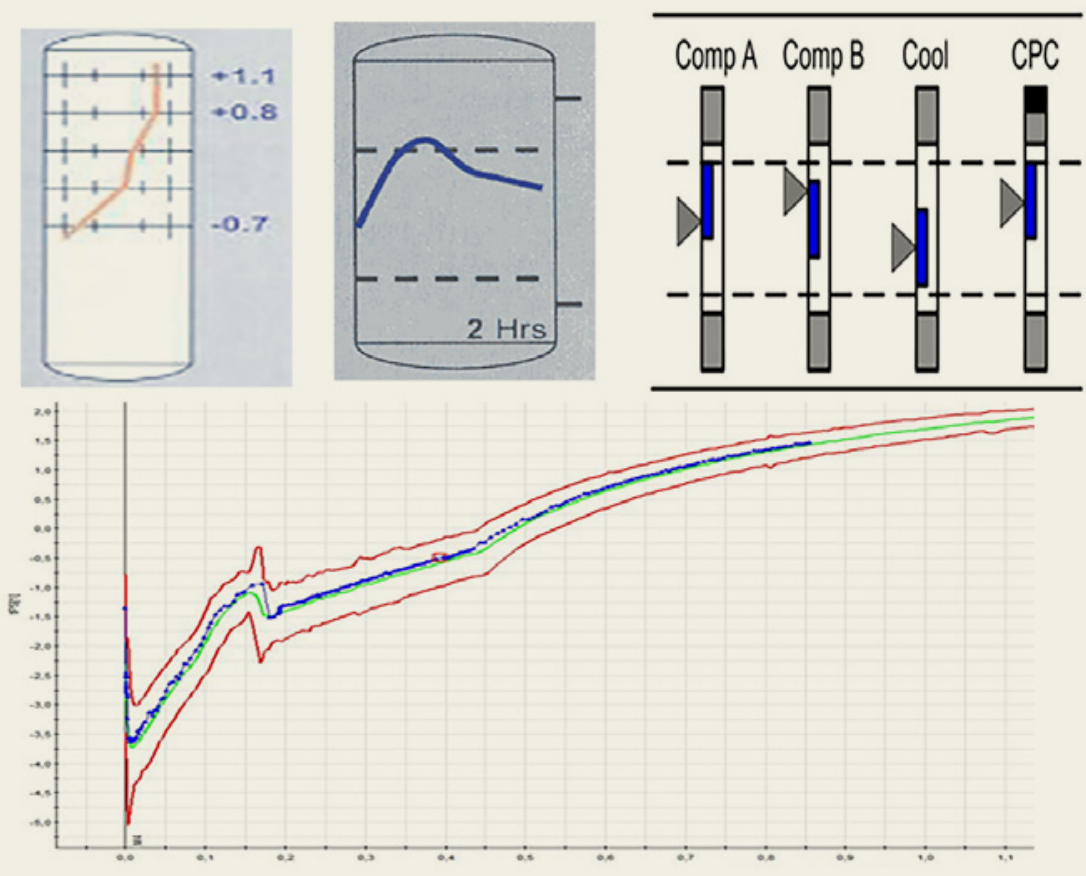

BILD 6: Analoge Darstellungen von Prozesszuständen einschließlich Akzeptanzbereichen

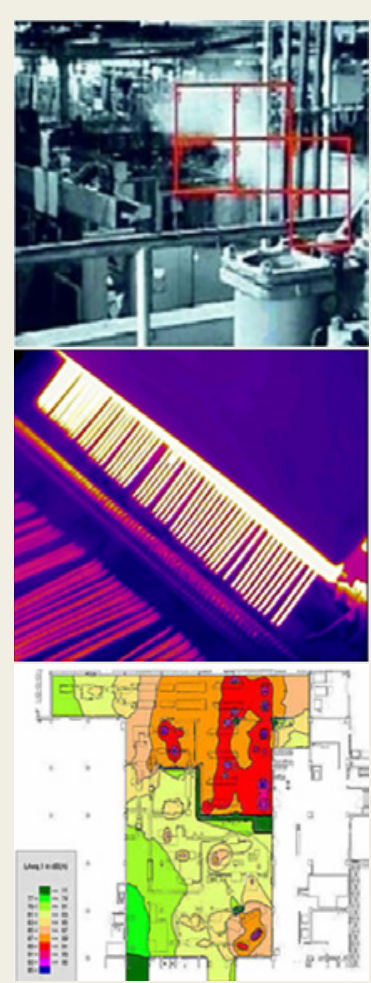

BILD 7:

Sensoren und Systeme zur räumlichen Überwachung von Anlagen lüberwachungskamera, Thermografie, Geräuschzustand)

besondere für kontinuierliche Prozesse besteht Entwicklungsbedarf für zielgruppengerechte PLS-Module, um die Komplexität für die Operateure zu reduzieren.

Bei der Regelgüte gibt es noch in vielen Anlagen deutlichen Optimierungsbedarf, um die ständig erforderlichen Interaktionen durch Operateure zu minimieren. Maßnahmen sind eine gezielte Sensor- und Aktorauswahl, ein Reglertuning sowie ein kontinuierliches Regelgüte-Monitoring, um Verschlechterungen zum Beispiel durch Aktorverschleiß, frühzeitig zu erkennen und rechtzeitig Gegenmaßnahmen einleiten zu können. Während bei kontinuierlich betriebenen Prozessen durch die anwendbare lineare Theorie die Lösungsansätze weitgehend klar sind, besteht bei nichtlinearen Batch-Prozessen noch weiterer Entwicklungsbedarf.

Anlagenintegrität: Die Anlagenintegrität ist unabhängig von der Anlagenautomatisierung durch die Überwachung des verfahrenstechnischen Prozesses und des Anlagenumfelds sicherzustellen. Dies umfasst unter anderem die frühzeitige automatische Erkennung von Kavitation, Leckagen oder Überhitzungen. Erforderlich ist, die im Rahmen von regelmäßigen Anlagenrundgängen über die menschlichen Sinneswahrnehmungen Sehen, Hören, Riechen erkannten Unregelmäßigkeiten sowie Gefahren durch integrale Sensoren zu erkennen und automatisch zu alarmieren. Die Basis hierfür können stark standardisierte und flächendeckend installierte oder mobile Videoüberwachungen, Thermografiekameras sowie Geräuschsensoren darstellen, vergleiche Bild 7.

Entwicklungsbedarf besteht für stark standardisierte Sensorcluster, mobile Inspektionseinheiten und Plattformen, mit denen derartige integrale Informationen zielgruppengerecht visualisiert und UnregelmäBigkeiten beziehungsweise Gefahren, beispielsweise auf Basis von Mustererkennungen, alarmiert werden.

\subsection{Aufwand und Nutzen}

Eine der ersten hochautomatisierten Anlagen der BASF ist der Antioxidanzien-Betrieb in Kaisten (Schweiz). Diese Anlage wird mit nur einem Operator gefahren und ist über Nacht und am Wochenende unbesetzt.

Kritische Prozessalarme werden an eine besetzte Nachbaranlage übertragen, von der aus die erforderliche Reaktion erfolgt. Maßnahmen gegen langsame Prozessdrifts werden in der Tagschicht ergriffen.

Der Erfolg fußt auf einem tiefen Prozessverständnis, klaren Zielen sowie einer konsequenten Implementierung und Instandhaltung. Realisiert ist diese hochau- 


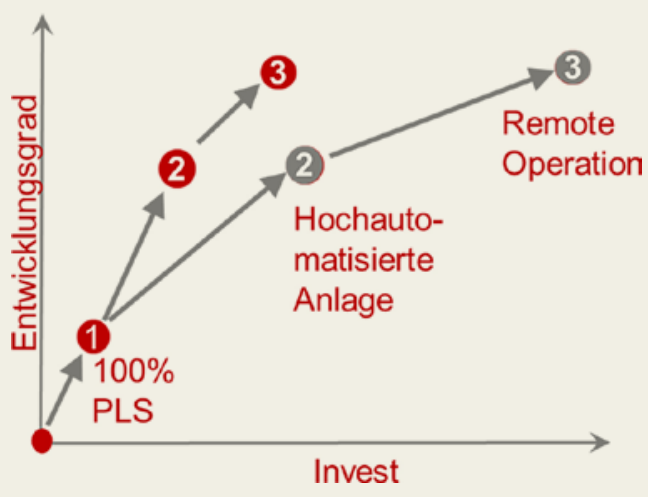

BILD 8: Reduzierung der Investitionskosten bei der Implementierung von hochautomatisierten beziehungsweise teilautarken Anlagen

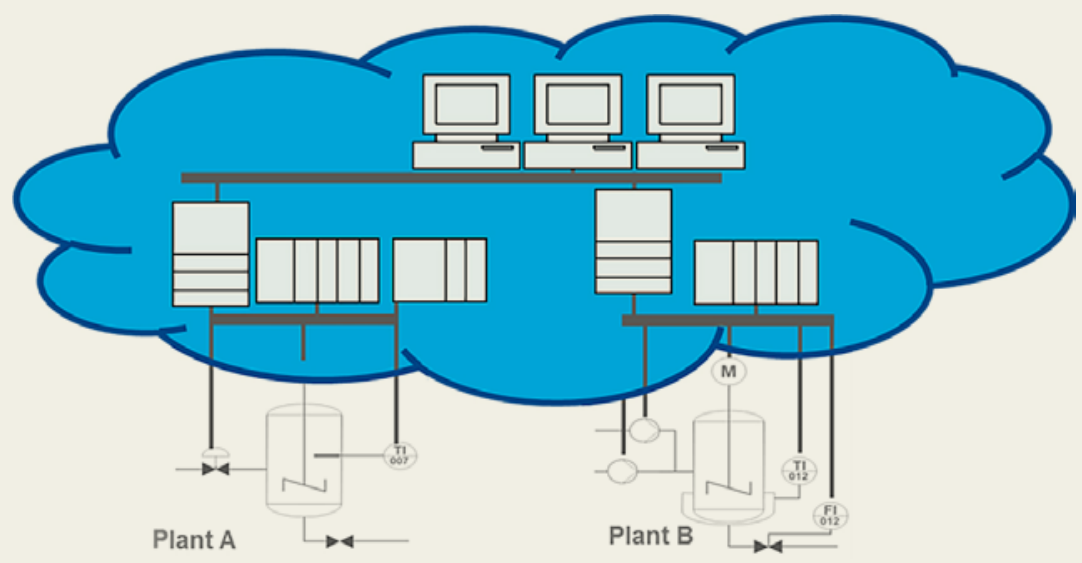

BILD 10: Das Prozessleitsystem in der Cloud

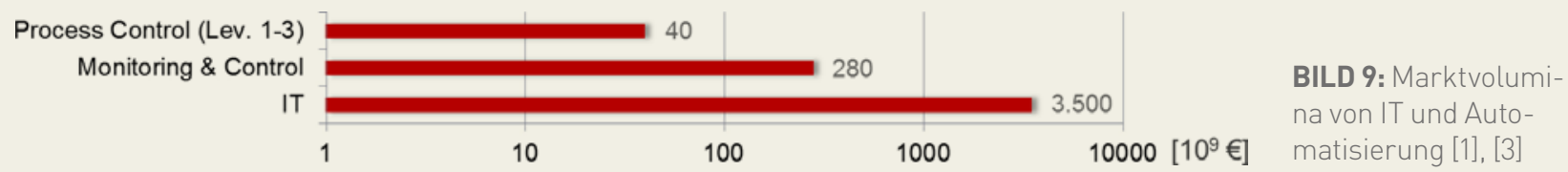

tomatisierte Anlage mit einem PLS aus dem Jahr 1994, was zeigt, dass derartige Lösungen ohne neueste Automatisierungstechnologie möglich sind. Allerdings war der Implementierungsaufwand sehr hoch. Die Anforderung an innovative Automatisierungstechnik ist daher, den Aufwand für derartige Anwendungen deutlich zu senken. Weiterer Entwicklungsbedarf besteht für eine Engineeringsystematik für Assistenzsysteme, siehe zum Beispiel [7].

Einbindung von Package Units: Lokale Bedienungen und Beobachtungen an Package Units sind offensichtlich für Remote Operation nicht möglich: Die komplette Bedienung und Beobachtung muss durchgängig über das PLS erfolgen. Der Entwicklungsbedarf hinsichtlich Plug and produce wurde durch die Namur bereits mehrfach betont.

Implementierungs-Roadmaps: Aufgrund der unterschiedlichen Historie und den langen Lebenszyklen des Equipments und der automatisierungstechnischen Komponenten kann die Einführung von Remote Operation in verfahrenstechnischen Anlagen unter wirtschaftlichen Gesichtspunkten nur schrittweise und häufig über Jahre hinweg erfolgen. Diese Schritte sind mit den anlagenspezifischen Bedarfen zu synchronisieren, da jeder Schritt eine eigene Wirtschaftlichkeit erfordert. Beispiele für anlagenspezifische Bedarfe sind
Kapazitätssteigerungen, ein Migrationsbedarf oder die Kompensation demografisch bedingter Abgänge.

Der erwartete Nutzen besteht in einer Reduktion variabler Kosten durch verringerte Rohstoff- und Energiekosten, einer Erhöhung der Anlagenverfügbarkeit durch die Vernetzung der Expertise sowie einer Reduktion der Fixkosten.

Anregung für die automatisierungstechnische Community: Zur Minimierung des Aufwands in den Projektteams bei der Einführung von Remote Operations wären Richtlinien und Checklisten mit den Themenfeldern Genehmigungssituation, Anlagensicherheit, Umweltschutz, Betreiberaspekte, Mitarbeiter-Qualifikation und Erfolgsfaktoren sehr hilfreich. Das Ziel kann es sein, für Hochschulen, Lieferanten und Anwender einen Mainstream der Entwicklung für die nächsten Jahre zu definieren und dadurch den Implementierungsaufwand für hochautomatisierte und teilautarke Anlagen zu senken, vergleiche Bild 8.

\section{SPANNUNGSFELD VON AUTOMATISIERUNG UND IT}

Industrie 4.0 ist die Initiative in Richtung einer Lösung. Aber was ist die Basis, die uns die Vielzahl neuer Lösungen ermöglichen soll? Wie in Abschnitt 1 dargestellt, sind es sehr häufig Produkte, Lösungen und auch die 
Methoden der IT. Durch die IT entsteht ein Technology Push für den Automatisierungsmarkt, und eine hohe Innovationsrate wird durch Mitzieheffekte forciert. Für den Weg vom Röhrenrechner zur SPS im Feld waren sehr viele Entwicklungen notwendig. Um nur einige zu Nennen: SMD-Technik, Miniaturisierung von Leiterbahnen, fortgeschrittene Netzwerktechnik und vieles mehr. Die wirklich großen Meilensteine auf dem Weg vom Röhrenrechner zur modernen SPS haben dabei zwei Punkte gemeinsam: Erstens, sie wurden nicht primär für die Automatisierung vorangetrieben sondern für die IT allgemein und zweitens, es waren gigantische Ressourcen notwendig, die nur noch die IT hat, siehe Bild 9.

Wenn Industrie 4.0 es erfordert, mehr Intelligenz in die Produktion und die umliegenden Prozesse wie Engineering zu bringen, so kann dies nur durch den vermehrten Einsatz von IT-Methoden funktionieren! Das bringt uns zu den Konsequenzen der aktuellen Weiterentwicklungen. Es findet ein Verschmelzen der Automatisierung mit der IT statt. Bisher ist es uns, zumindest weitestgehend erfolgreich, gelungen die Hard- und Software der IT in Leitsysteme zu integrieren, auch wenn das zu Beginn leidvolle Erfahrungen mit sich brachte und auch die ein oder andere Anpassung von Service- und Maintenancekonzepten sowie den entsprechenden Know-how-Aufbau erforderte. Die positiv empfundenen Aspekte sind die günstige Marktverfügbarkeit der Produkte und die existierenden Standards, die sich wegen des Käuferverhaltens und der hohen Innovationsrate schnell herauskristallisieren.

Hier liegt die Rolle der Automatisierung in der Gestaltung, konkret zum Beispiel in der Beschreibung der Anforderungen an Netzwerke, Verfügbarkeiten, Lebenszyklen- und Servicekonzepte.

\section{REFERENZEN}

[1] European Commission, Final Report of the Study SMART, 2007

[2] Google Public Data: WeltentwicklungsindikatorenFruchtbarkeitsrate der Welt 1960-2012, http://www.google.de/publicdata/explore?ds=d5bncppjo f8f9_\#!ctype $=l \&$ strail $=$ false $\& b c s=d \& n s e l m=h \& m$ et_y=sp_dyn_tfrt_in\&scale_y=lin\&ind_=false \&rdim $=r e$ gion\&ifdim=region \&tdim=true \&hl=de\&dl=de\&ind=false

[3] Gartner Worldwide IT Spending Forecast, http://www.gartner.com/technology/research/ it-spending-forecast, 2014

[4] 5 G Lab Germany, 5glab.de, 2014

[5] Geschäftsbericht Linde 2012, Linde Group, München, 2012

[6] European Industrial Gases Association (EIGA, www. EIGA.com): IGC Document 132/05/E „Unmanned Air Gas Plants - Design and Operation“, Brüssel, 2005

[7] Obst M.; Doherr F.; Urbas L.: Wissensbasiertes Assistenzsystem für modulares Engineering. at Automatisierungstechnik 61(2), S. 103-108, 2013. DOI:10.1524/auto.2013.0011
Sehen wir uns ein Beispiel an: Wenn Themen wie Virtualisierung und Weiterentwicklungen der Netzwerktechnik kombiniert werden, scheinen Leitsysteme in der Cloud geradezu unumgänglich, siehe Bild 10, weil die Idee einfach zu offensichtlich ist, nachdem beispielsweise die MES-Systeme bereits in Rechenzentren beheimatet sind. Hier muss die Botschaft ganz klar sein und unsere Interessengemeinschaft sich ganz eindeutig verhalten: Mitgestalten soviel es nur geht!

MANUSKRIPTEINGANG 08.12.2014

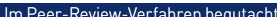

\section{AUTOREN}

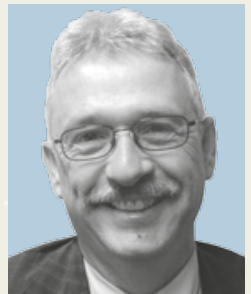

Dr.-Ing. JOACHIM BIRK

(geb. 1961) ist seit seiner Promotion über nichtlineare Systemdynamik und Regelungstechnik in der BASF SE tätig. Er leitet das Fachzentrum Automatisierungstechnik in Ludwigshafen und verantwortet die Fachgebiete Prozessleitsystem-Technologie, Validierungspflichtige Automatisierungslösungen, Industrielle Kommunikationstechnik, Advanced Process Control (APC) und Manufacturing Execution Systems (MES).

BASF SE,

GTG/ED - L440, D-67056 Ludwigshafen,

Tel. +49 (0) 6216055284 ,

E-Mail: joachim.birk@basf.com

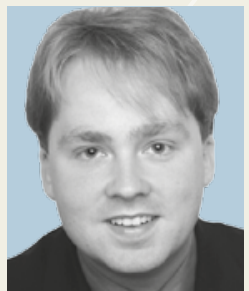

\section{Dr. MICHAEL KRAUSS}

(geb. 1984) ist seit seiner Promotion in theoretischer Physik 2010 im Fachzentrum für Automatisierung der BASF SE in Ludwigshafen tätig. Seit 2013 leitet er ein Fachgebiet für Prozessleitsysteme mit dem Fokus auf Automatisierungsprojekten. Im Januar 2014 übernahm er die Leitung des NAMUR-Arbeitskreises 2.1 „PLS und SPS“.

BASF SE,

GTG/ED - L440, D-67056 Ludwigshafen,

Tel. +49 (0) 6216046694 ,

E-Mail: michael.krauss@abasf.com 